\title{
Inexpensive circuit for the measurement of capture cross section of deep level defects in semiconductors
}

\author{
C. V. Reddy, S. Fung, and C. D. Beling \\ Department of Physics, The University of Hong Kong, Hong Kong
}

(Received 3 May 1996; accepted for publication 15 August 1996)

\begin{abstract}
A simple and inexpensive circuit to facilitate the direct measurement of capture cross section, when synchronized with a deep level transient spectroscopy system, is described. It avoids the most commonly encountered problem of loading and distortion of the bias (trap filling) pulses of nanosecond duration in the capture cross-section measurement. The capacitance meter, whose internal circuitry is responsible for the distortion, is connected and disconnected from the rest of the apparatus with the help of simple and low-cost reed relay switches featuring high operating speed and low contact resistance. Sharp bias pulses as small as $30 \mathrm{~ns}$ can successfully be applied to the sample with no observable distortion. Finally, a representative measurement is shown to demonstrate the simplicity and high performance of the circuit. (C) 1996 American Institute of Physics. [S0034-6748(96)03311-4]
\end{abstract}

\section{INTRODUCTION}

The carrier capture cross section of a semiconductor deep level defect contains useful information on the nature of the defect. This parameter is directly determined by measuring the capture time constant $\tau_{c}$ of the deep level

$$
\tau_{c}=1 / \sigma_{n} v_{n} n,
$$

where $\sigma_{n}$ is the cross section, $v_{n}$ is the thermal velocity of electrons, and $n$ is the free carrier concentration at the measurement temperature. $\tau_{c}$ is often measured by monitoring the deep level transient spectroscopy (DLTS) peak height $\left[S\left(t_{p}\right)\right]$ as a function of filling (bias) pulse width $\left(t_{p}\right),{ }^{1}$ the slope of the plot $\ln \left[1-S\left(t_{p}\right) / S\left(t_{\infty}\right)\right]$ versus $t_{p}$ giving $\left(\tau_{c}\right)^{-1}$, where $S\left(t_{\infty}\right)$ is the saturated DLTS peak height. While the above plot is usually found to be nonlinear due to the different capture rates of the free carrier tail extending into the depletion region, the initial part of the plot, which is fairly linear, gives a reliable estimate of the $\tau_{c} .{ }^{2}$ By measuring the cross section for both electrons and holes, the generation or recombination behavior of the defect can easily be determined. Furthermore, by measuring the cross section at different temperatures, one can determine the capture barrier or the activation energy $\Delta E$, which with the Franck-Condon shift $\left(d_{\mathrm{FC}}\right)$ parameter, obtainable through optical techniques, allows a configuration coordinate diagram to be constructed. Such a diagram gives a helpful visualization of the electronphonon interaction, and can reveal lattice relaxations that occur on charge carrier capture. ${ }^{3}$ One such example for large lattice relaxation (LLR) is the DX center observed in $\mathrm{AlGaAs},{ }^{4} \mathrm{GaAsP},{ }^{5}$ and AlGaInP, ${ }^{6}$ which exhibits persistent photoconductivity. Capture cross section is thus becoming one of the most important parameters to be determined in order to understand the physical nature of a defect.

\section{CIRCUIT DETAILS}

In the measurement of capture cross section, the experimental difficulty lies in the successful application of the short duration trap filling pulses to the sample. This is because the pulse gets distorted for two main reasons. The first is due to the overloading effect by the internal circuitry of the capacitance meter while the second problem is due to the reflection of the bias pulse as a result of impedance mismatching between the sample and the pulse generator. The latter can be solved by shortening the cable length between the sample and pulse generator as much as possible. However, the first problem can only be solved first by disconnecting the capacitance meter during the application of the bias pulse and then connecting back to the sample to monitor the emission transient immediately after the bias pulse. This process is shown schematically in Fig. 1(a). The present article describes a simple circuit which we have recently constructed that facilitates the direct measurement of capture cross section, in a DLTS spectrometer. Although the circuit is designed to work in conjunction with a home-built spectrometer, ${ }^{7}$ it is sufficiently general for its easy adaptation to any other DLTS system.

The circuit, shown in Fig. 1(b), is constructed using double pole normally open (DPNO) and single pole change over (SPCO) reed relay switches, logic gates, and transistors. The miniature relays were procured from Radio Spares, UK (Model Nos. 349-248 and 350-541, respectively), and are directly PCB mountable. Their main features are high operating speed, low bounce time, low contact resistance, and a large number of operations. The DPNO switch is normally open when no power is supplied and closes when it is powered, while the SPCO changes its switch position from one to the other, as shown in Fig. 1(b), when it is powered. The closing and opening of the switches, the consequent connection and disconnection of the capacitance meter are synchronized with the DLTS measurement sequence through the rate-window timing circuitry (RSC unit) of the spectrometer, whose details are discussed elsewhere. ${ }^{7}$ As mentioned in Ref. 7, the sequence of events that take place in a DLTS 

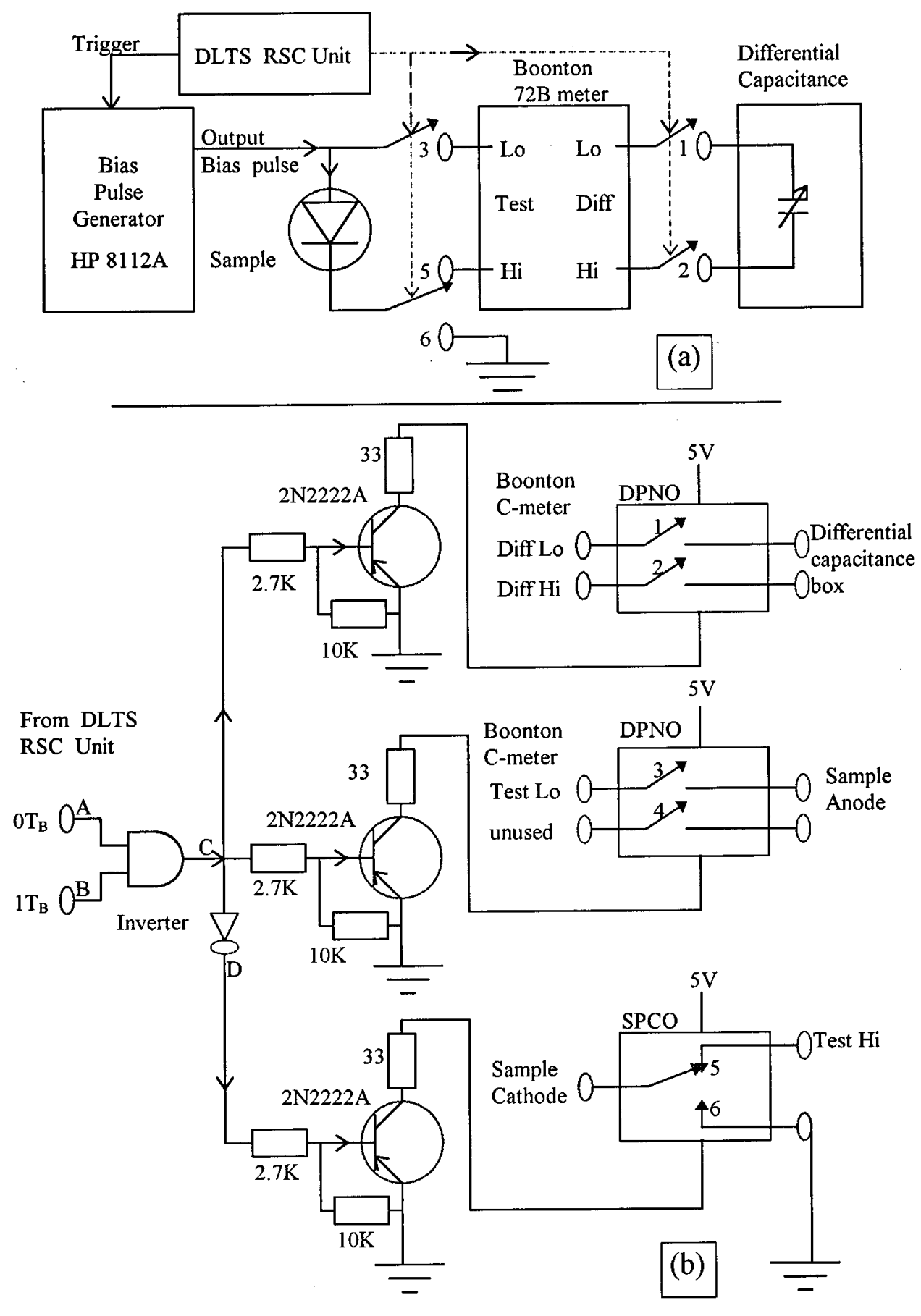

FIG. 1. (a) The schematic and (b) the circuit diagrams of the DLTS experimental setup for capture cross-section measurement. Relay switch numbers 1 to 6 in (a) are identical to those in (b).

measurement are controlled by the time sequenced TTL outputs of the counter 74LS154, which are labeled as $0 T_{B}$, $1 T_{B}, \ldots, 15 T_{B}$. The measuring sequence starts by triggering the pulse generator at $0 T_{B}$, sampling the capacitance transient at rate-window timings $t_{1}\left(3 T_{B}\right)$ and $t_{2}\left(13 T_{B}\right)$, followed by base line correction at $15 T_{B}$. However, only the first two outputs, $0 T_{B}$ and $1 T_{B}$, which are available on the front panel of the spectrometer, are utilized in this circuit in order to achieve the synchronization. These two outputs are fed to an AND gate, whose output is finally connected to the base of a transistor. A $\operatorname{Lo}(0 \mathrm{~V})$ pulse at the base drives the transistor into cut-off stage while a $\mathrm{Hi}(5 \mathrm{~V})$ pulse drives the transistor into saturation. Thus the transistor is simply used as a switch which rather connects or disconnects the reed relay's ground point from that of the chassis. A direct connection of the logic pulse to the reed relay in order to open or close the switch may result in damaging the time-base circuitry of the spectrometer.

To describe the sequence of events that take place in this experiment, let us start with $0 T_{B}$, which goes from $\mathrm{Hi}(5 \mathrm{~V})$ 


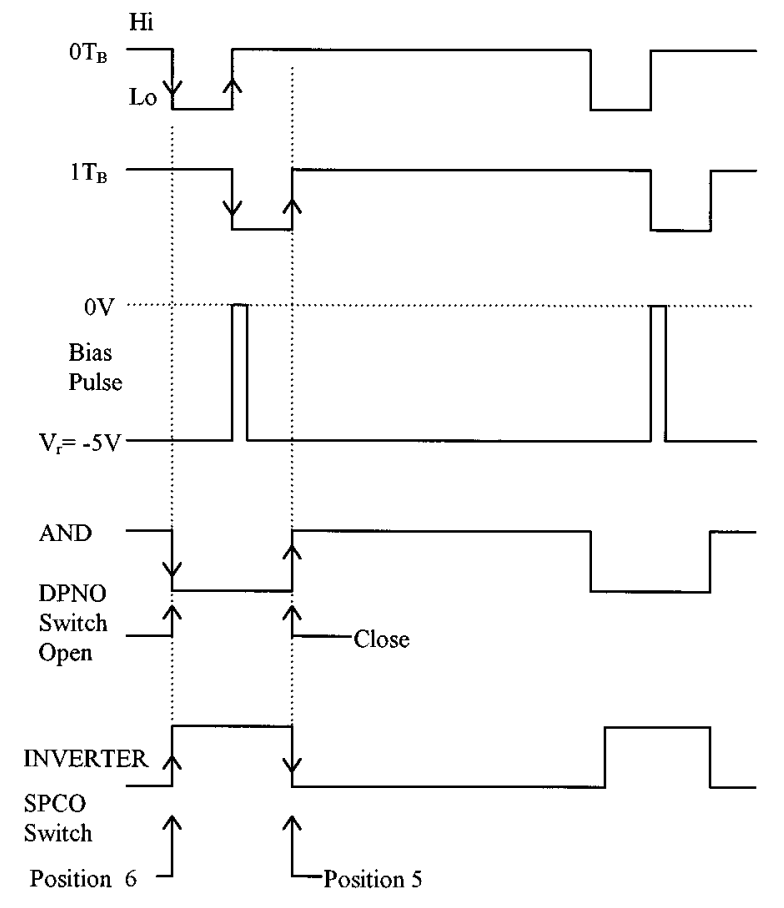

FIG. 2. Timing diagram showing the synchronization between the DLTS time sequence pulses $0 T_{B}, 1 T_{B}$, the trap filling pulse, and the relay activation pulses.

to $\operatorname{Lo}(0 \mathrm{~V})$, as shown in Fig. 2, when the measurement is started. This falling edge drives the AND output to Lo, which further drives the transistor into cut-off stage and thus the reed relay switch is opened. As a result of this, the capacitance meter is disconnected from the sample and the rest of the spectrometer. Now, the bias pulse is applied to the sample by triggering the pulse generator on the rising edge (Lo to $\mathrm{Hi}$ ) of $0 T_{B}$. It is important to note here that when the $0 T_{B}$ goes from Lo to $\mathrm{Hi}$, then $1 T_{B}$ goes from $\mathrm{Hi}$ to Lo simultaneously. Thus the capacitance meter is still disconnected since AND gate output remains Lo, as it was before. The meter is connected back to the sample in order to monitor the capacitance transient at the rising edge of the $1 T_{B}$. Thus, sharp bias pulses can be applied to the sample with a very minimum distortion. Since the sample (cathode) is usually grounded through the capacitance meter, there exists a problem in applying the trap filling pulse when the meter is disconnected, since the sample is left ungrounded. To circumvent this problem, the SPCO switch is used to ground the sample during the bias pulse, as shown in Fig. 1.

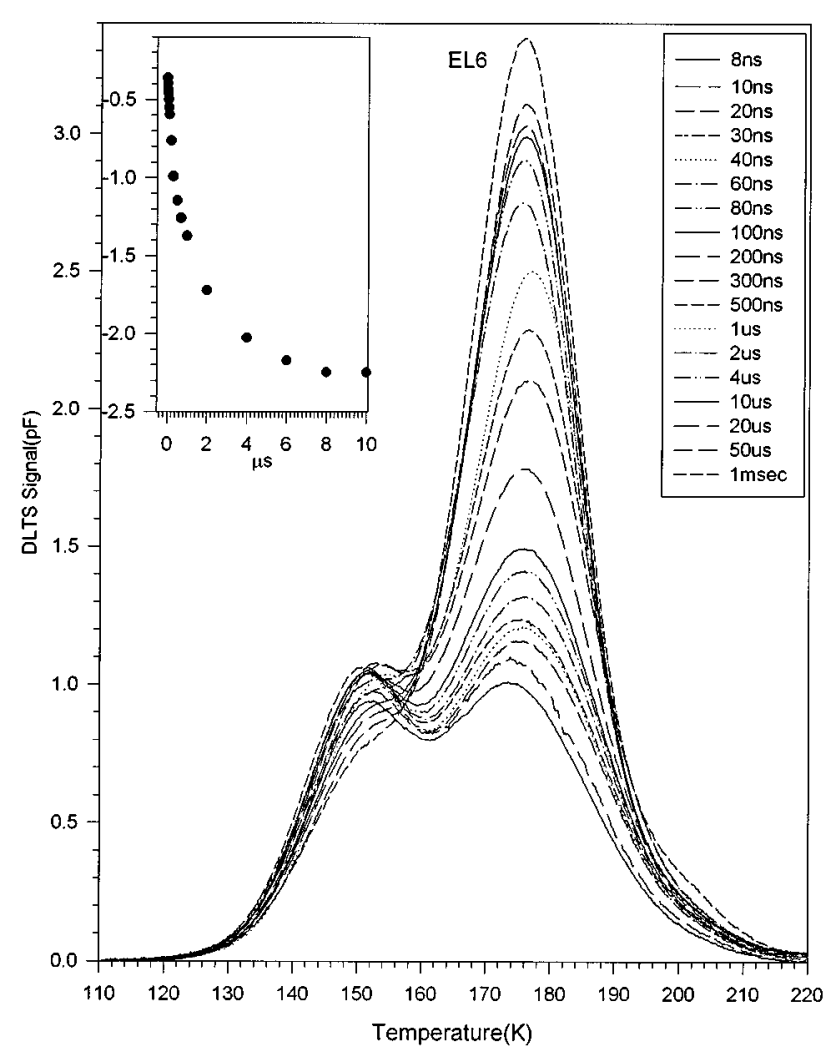

FIG. 3. A typical capture cross-section measurement taken on the EL6 defect in $n$-GaAs.

\section{RESULTS}

The capture cross-section measurement taken on the EL6 defect in $n$-GaAs is shown in Fig. 3. The inset shows the plot of $\ln \left[1-S\left(t_{p}\right) / S\left(t_{\infty}\right)\right]$ versus $t_{p}$. By substituting the relevant values in Eq. (1), the cross section $\left(\sigma_{n}\right)$ for the EL6 defect is determined as $1.17 \times 10^{-17} \mathrm{~cm}^{2}$ at $177 \mathrm{~K}$.

${ }^{1}$ J. A. Borsuk and R. M. Swanson, J. Appl. Phys. 52, 6704 (1981).

${ }^{2}$ H. Indusekhar and V. Kumar, Phys. Status Solidi A 95, 269 (1986).

${ }^{3}$ J. C. Bourgoin, H. J. von Bardeleben, and D. Stièvenard, J. Appl. Phys. 64, R65 (1988).

${ }^{4}$ D. V. Lang, R. A. Logan, and M. Jaros, Phys. Rev. B 19, 1015 (1979).

${ }^{5}$ E. Calleja, F. J. Sanchez, E. Muñoz, E. Vigil, F. Omnés, P. Gibart, J. M. Martin, and G. Gonzalez Dìez, Phys. Rev. B 53, 7736 (1996).

${ }^{6}$ J. Mäkinen, T. Laine, J. Partanen, K. Saarinen, P. Hautojärvi, K. Tappura, T. Hakkarainen, H. Asonen, M. Pessa, J. P. Kauppinen, K. Vänttinen, M. A. Paalanen, and J. Likonen, Phys. Rev. B 53, 7851 (1996).

${ }^{7}$ C. V. Reddy, S. Fung, and C. D. Beling, Rev. Sci. Instrum. 67, 257 (1996). 